\title{
ÄRZTEPORTAL
}

\section{Die Deutschen mögen ihren Doktor}

Internetportale, in denen Patienten ihren Doktor benoten, werden von Ärzten mit einem gewissen Misstrauen beäugt. Die Auswertung von ca. 32000 Bewertungen im Ärzteportal jameda zeigt jetzt, dass die Bundesbürger ihren Ärzten gegenüber doch recht wohlwollend gesinnt sind. Behandlungserfolg, Freundlichkeit, Aufklärungsumfang und die Zeit, die der Arzt sich für seine Patienten genommen hatte, wurden mit Schulnoten von eins bis sechs benotet. Die niedergelassenen Ärzte erhielten von den Kassenpatienten eine glatte 2 und von den Privatpatienten eine 1,8. Lediglich in Berlin weicht die Zufriedenheit der Privatpatienten signifikant von der der Kassenpatienten ab: Privat erhalten die Berliner Doktores eine 1,6, auf Kasse eine 2,3.

Pressemeldung jameda

\section{OPERATION}

\section{Rauchstopp beschleunigt Heilung}

Raucher, die ungeplant operiert werden müssen, sollten zum Nikotinentzug motiviert werden. Der Verzicht auf die Zigarette beschleunigt nämlich den Heilungsprozess und reduziert die Komplikationsrate. Bisher war der Nutzen einer solchen Maßnahme nur im Vorfeld von elektiven Operationen belegt. Dass sich der Rauchstopp auch noch postoperativ günstig auswirkt, zeigt nun eine randomisierte Studie mit 105 Patienten, bei denen eine Fraktur chirurgisch behandelt werden musste. J Bone Joint Surg 2010;92:1335-1342

\section{ÜBERGEWICHT}

\section{Jeder zweite} Erwachsene zu dick

In Deutschland ist mittlerweile jeder zweite Erwachsene (51\%) übergewichtig (BMI > 25). Dies ergab die MikrozensusZusatzbefragung 2009. Männer sind häufiger dick als Frauen (60\% vs. 43\%). Wer als Single lebt, achtet mehr auf sein Gewicht als Verheiratete: $56 \%$ der ledigen Männer und $69 \%$ der ledigen Frauen sind normalgewichtig. Untergewicht (BMI < 18,5 ) tritt deutlich seltener auf (Frauen 3\%, Männer 1\%), von den Frauen unter 25 sind aber 9-13\% zu dünn.

Pressemitteilung des Statistischen Bundesamts Deutschland vom 2.6.2010

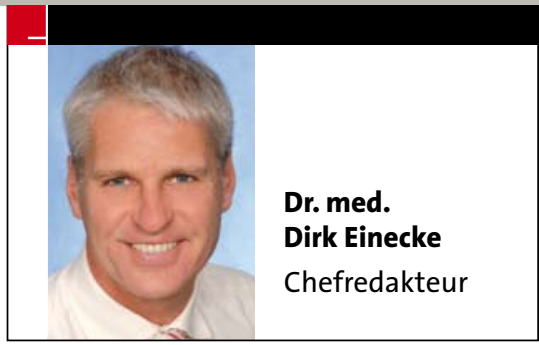

\section{DIABETESTHERAPIE}

\section{Basalinsulin-Analoga weiter Kassenleistung}

Die lang wirksamen Insulinanaloga bleiben einstweilen zu Lasten der Krankenkassen verordnungsfähig, auch wenn sie mehr kosten als die Humaninsuline. Eine Verordnungseinschränkung, die der Gemeinsame Bundesausschuss im März beschlossen hatte, hat das Bundesgesundheitsministerium nicht genehmigt. Vielmehr soll der Bundesausschuss jetzt klären, ob durch den Einsatz von lang wirksamen Insulinanaloga regelhaft Unterschiede bei der Inanspruchnahme ärztlicher Behandlung oder anderer Leistungen entstehen.

\section{ANTIDEPRESSIVA \\ Kataraktgefahr unter SSRI}

Serotonin-Wiederaufnahmehemmer (SSRI) können das Kataraktrisiko erhöhen. Das lässt eine Fall-Kontroll-Studie mit 19000 Kataraktpatienten und 190000 Kontrollen vermuten. SSRI-Anwender hatten ein um $15 \%$ erhöhtes Katarkatrisiko. Am höchsten war das Risiko unter Fluvoxamin, Venlafaxin und Paroxetin. Ob der Effekt nur bei bestimmten SSRI auftritt oder ob er klassenspezifisch ist, konnte nicht mit letzter Sicherheit festgestellt werden, da die Zahl der Probanden, die andere SSRI als die genannten einnahmen, zu gering war.

Ophtalmology, 2010;DOI:10.1016/ j.ophta.2009.11.042 